\title{
Restoration of Smile And Function in Partially Edentulous Patient With worn out Anterior Dentition
}

\author{
Dr. Viram Upadhyaya ${ }^{1}$,Dr. Aman Arora ${ }^{2}$,Dr. Prachi Jain ${ }^{3}$,Dr. Nitesh chopra ${ }^{4}$ \\ ${ }^{I}$ Reader, Department of Prosthodontics, J.N.Kapoor DAV(C) Dental College, Yamunanagar, 135001 \\ ${ }^{2}$ Head of Department, Department of Prosthodontics, J.N.Kapoor DAV $(C)$ \\ Dental College, Yamunanagar, \\ ${ }^{3}$ Post graduate student, Department of Prosthodontics, J.N.Kapoor DAV $(C)$ \\ Dental College, Yamunanagar, \\ ${ }^{4}$ Post graduate student, Department of Prosthodontics, J.N.Kapoor DAV $(C)$ \\ Dental College, Yamunanagar,
}

\begin{abstract}
Altered vertical dimension can have a detrimental effect on the functional integrity of dental arches and the associated structures as well as on the aesthetics of the patient. This paper presents the prosthodontic management where aim was to regain the lost vertical dimension in a partially edentulous patient with mutilated anterior dentition. The treatment involved use of both fixed and removable prosthesis and resulted in both functional and esthetic improvement in the patient.
\end{abstract}

Keywords: Rehabilitation, mutilated dentition, partial edentulism

\section{Introduction}

Restoration of the lost vertical dimension in a patient with collapsed bite is a challenging task as it has to be done in such a way that it does not interfere with the neuromuscular coordination of the temporomandibular joint (TMJ) while simultaneously fulfilling the patient's requirement of function and aesthetics ${ }^{1}$. Careful treatment planning using fixed or removable prosthesis is required to manage such cases to get a favourable prognosis. This article presents a case in which a multidisciplinary approach has been undertaken for rehabilitation in a partially edentulous patient with compromised remaining dentition both functionally and aesthetically.

\section{Case Report}

A 52 year old male patient reported to our Department with chief complaint of difficulty while mastication and unaesthetic appearance of the front teeth. He had a history of night grinding. The intraoral examination revealed missing 13,14,15,16,17,18,23 and 24 in the maxillary arch with crowns wrt 25,26 and 27. In the mandibular arch, 36, 37 and 38 were missing with crowns wrt 34,35,45,46 and 47. His anterior teeth were severely attrited with crossbite relationship. (Figure 1)

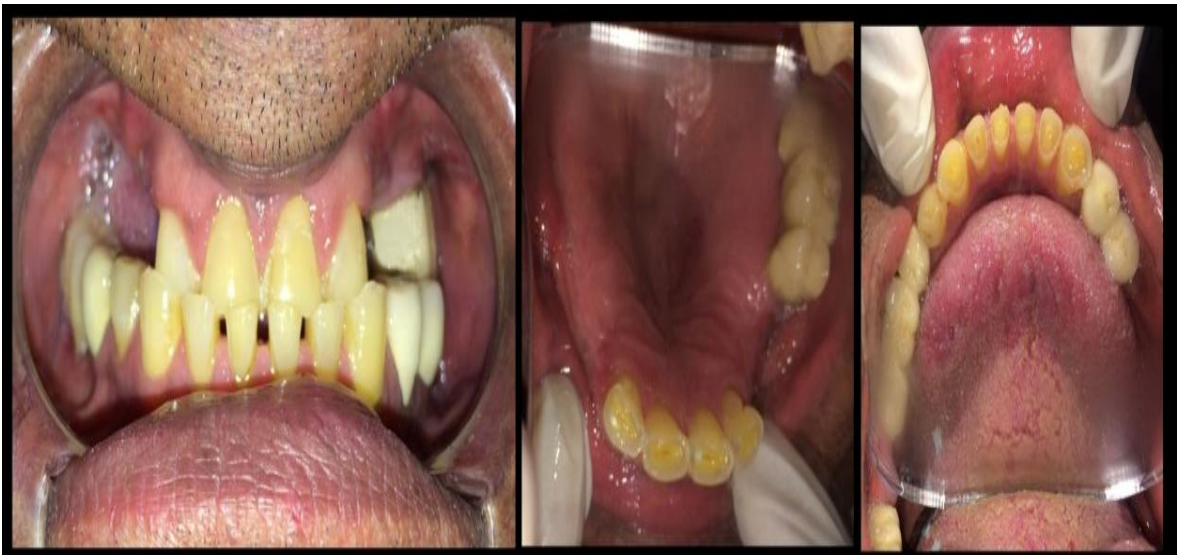

Fig 1: Pre rehabilitation intraoral view

Extraoral examination revealed an obvious overclosure with loss of lip fullness and drooping of the corners of the mouth indicative of loss of vertical dimension. Clinical analysis revealed a freeway space of 8mm. (Figure 2) 


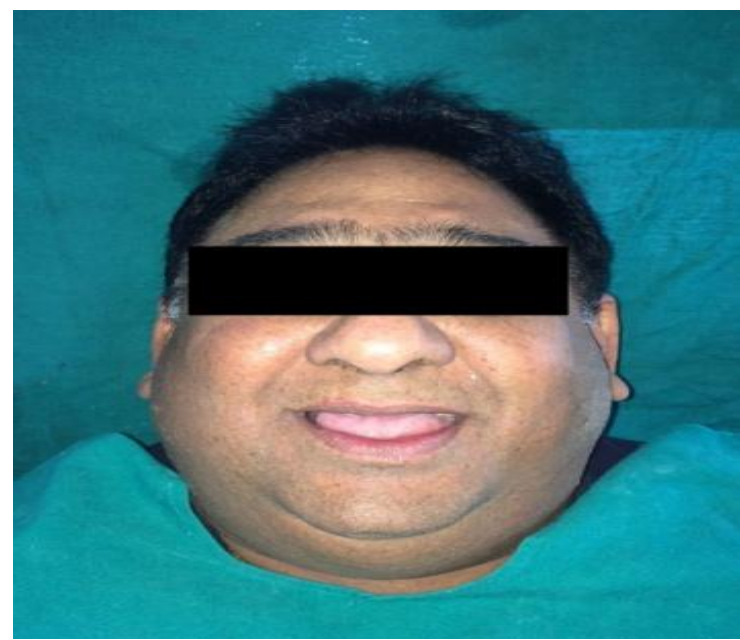

Fig 2: Pre rehabilitation extraoral view

Treatment protocol: To restore the lost vertical dimension, a $4 \mathrm{~mm}$ increase of vertical dimension was planned. The anterior attrited teeth were to be restored with porcelain fused to metal (PFM) crowns and posterior edentulous area was planned to be restored with removable flexible partial denture.

Procedure: Maxillary and mandibular diagnostic impressions were made and a soft occlusal splint of $4 \mathrm{~mm}$ was fabricated and given to the patient for a period of one month to allow the muscles and TMJ to accommodate to the new vertical dimension. At the recall visit, as the patient was found to be comfortable with raised vertical dimension of occlusion, it was decided to proceed according to the planned treatment.

First, root canal treatment was performed on 11,12,21,22,31,32,33,41,42,43 and 44. Impressions of both the arches were made in irreversible hydrocolloid impression material. The casts were mounted on a semi adjustable articulator using facebow (Figure 3).
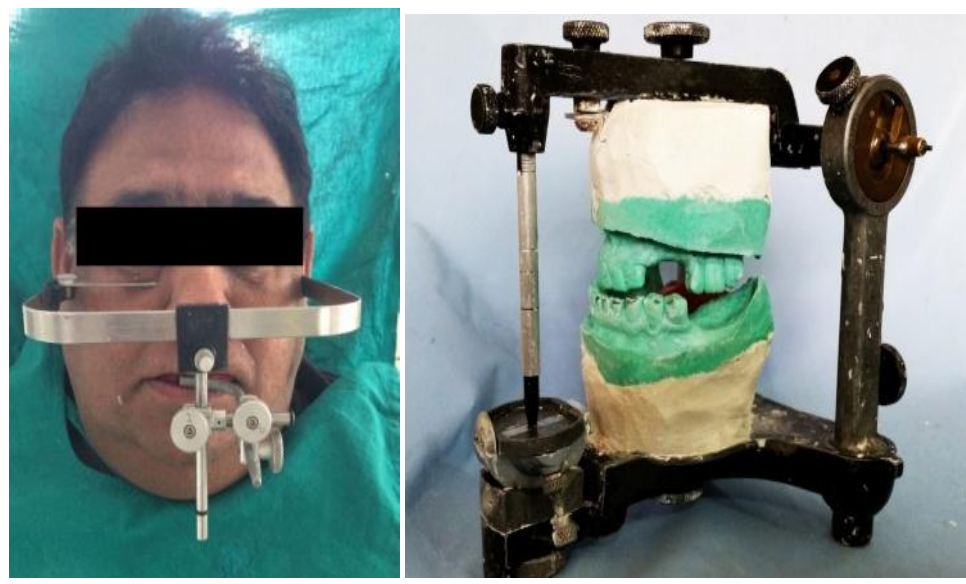

Fig. 3: Mounted casts on the articulator using facebow

Mounting was done at increased vertical dimension with the help of interocclusal record made using putty consistency of addition silicone. Diagnostic wax up was done in such a way so as to correct both the horizontal and vertical relation of the anterior teeth (Figure 4).

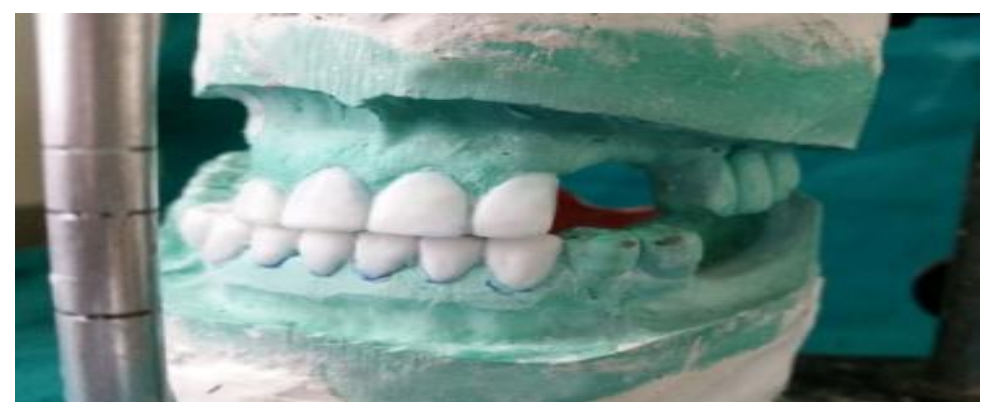

Fig. 4: Mounted casts on the articulator 
Crown preparation was carried out as per the protocols of the conventional PFM crown. To correct the anterior crossbite, greater reduction was done on the lingual aspect of maxillary teeth and labial aspect of mandibular teeth (Figure 5). After retraction, final impression was made in putty and light body polyvinyl siloxane impression material using double step technique (Figure 6). They were poured in die stone. Provisional restoration was fabricated using direct technique by making putty index of the diagnostic wax up (Figure 7).

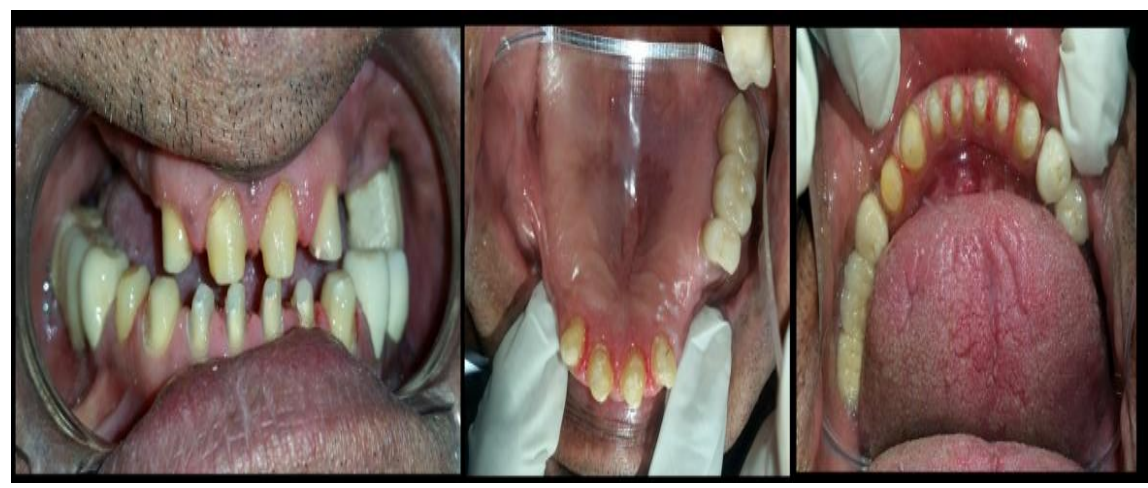

Fig. 5: Tooth preparation

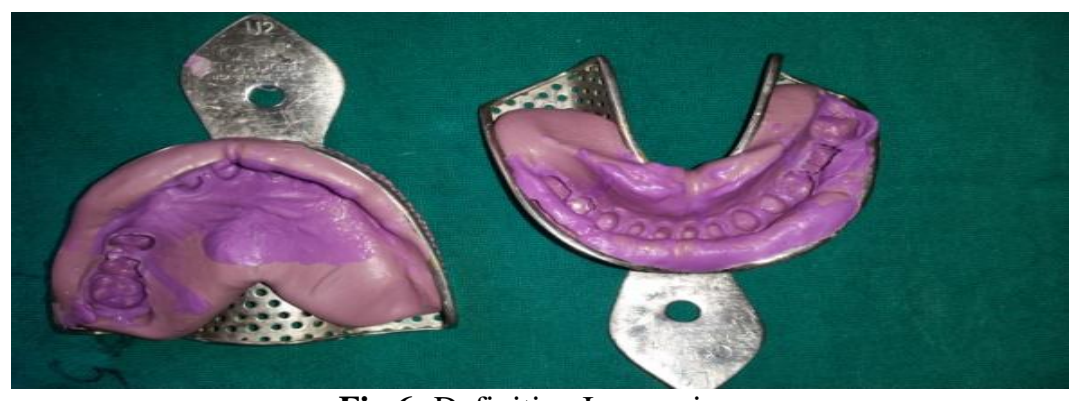

Fig.6: Definitive Impression

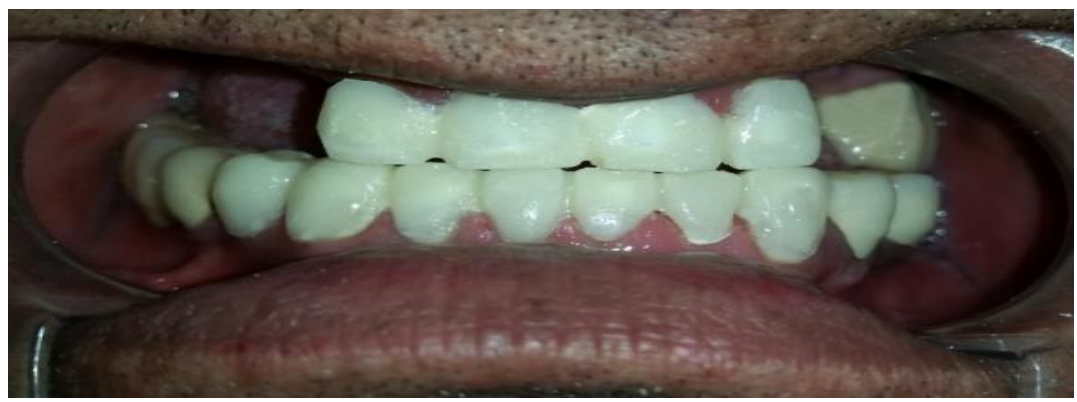

Fig. 7: Provisional restoration

Thereafter, wax pattern was fabricated on the cast retrieved and casting of the wax pattern was done followed by finishing. Metal trial was done in the patient's mouth (Figure 8). After verifying the fit of the prosthesis, ceramic build up was done. The final prosthesis was cemented using Glass inomer luting cement.

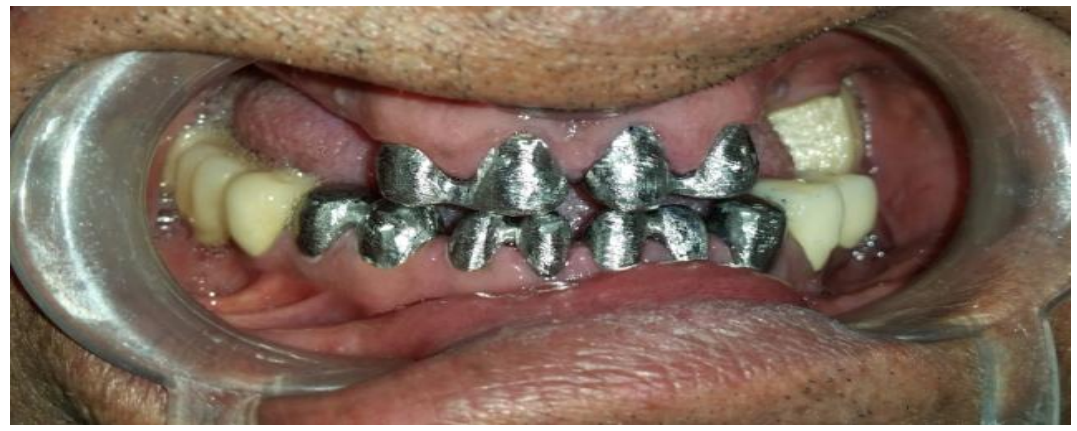

Fig. 8: Metal trial 
To fabricate the removable prosthesis, impressions were made and custom tray was fabricated on the cast retrieved. Border moulding was performed using low fusing impression compound and secondary impression was made with zinc oxide eugenol impression material. On the master cast, record base and occlusal rims were fabricated and jaw relations were recorded. Teeth setting and trial was then done followed by processing of the denture (Figure 9). The denture was inserted and post insertion instructions were given to the patient.

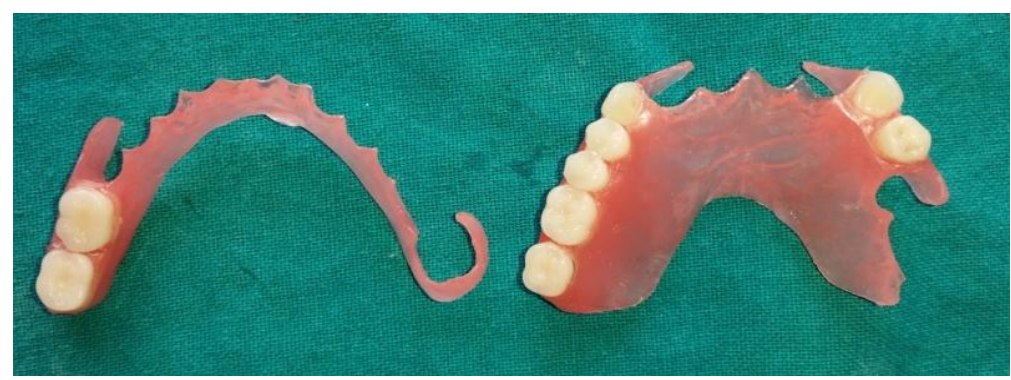

Fig. 9: Removable flexible partial denture

As the patient had the habit of night grinding, he was given a night guard to protect the crowns and enhance the success rate of our treatment. At the recall visit, the patient did not report with any discomfort and he was found to be happy with the aesthetics and function of the prosthesis (Figure 10 and 11).

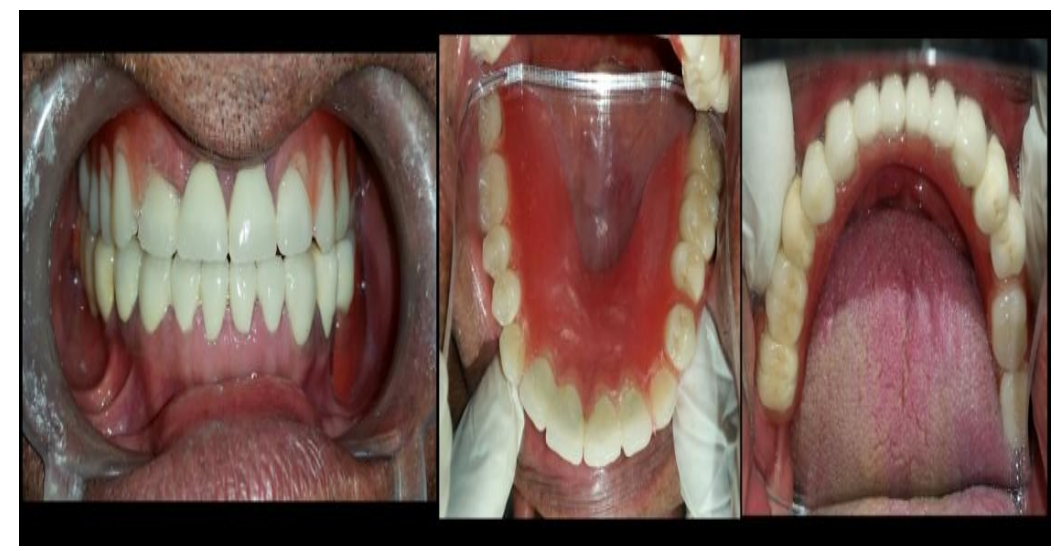

Fig. 10: Post rehabilitation intraoral view

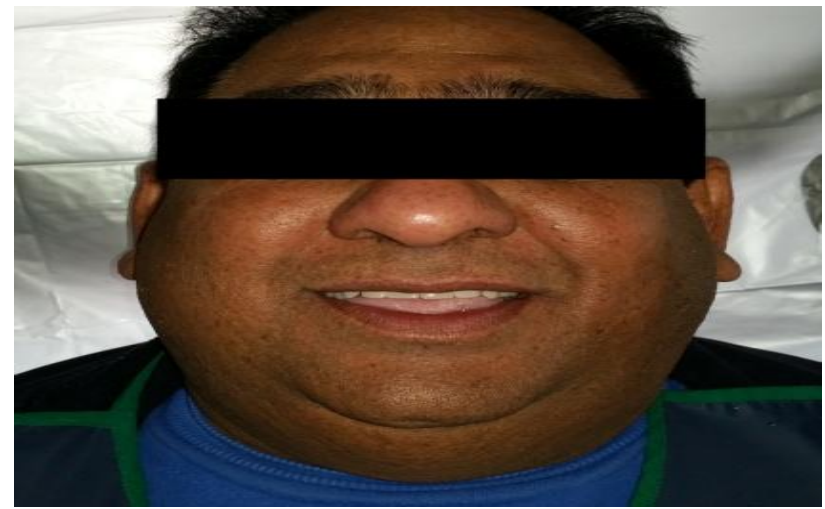

Fig. 11: Post rehabilitation extraoral view

\section{Discussion}

Goal of dentistry is to increase the lifespan of the dentition ${ }^{2}$. Normal physiological wear of the dentition usually does not require correction but excessive occlusal wear can lead to occlusal disharmony, esthetic disfigurement and loss of VDO. Therefore, restorative intervention to correct such condition is required.

In the present case report, the patient presented with complaint of inability to properly chew food and unaesthetic appearance. On examination excessively worn down dentition along with loss in VDO was seen. After proper evaluation, treatment plan was formulated which included use of occlusal splint to restore the lost vertical dimension and patient was kept in an observational period of one month so that he can adapt to the 
altered VDO. PFM crowns were fabricated for the anterior mutilated dentition along with flexible RPD to restore the edentulous area. The patient was found to be fully satisfied and much more confident at the end of the treatment.

\section{References}

[1]. Chidambaram R, Grover M, Veeravalli PT. Full mouth rehabilitation of a worn out dentiotion using multidisciplinary approach. J Orofacial Res Jan Mar 2013; 3 (1): 54-56.

[2]. Rathod AM, Shetty OK, Gharat SR, Dsouza R. All Zirconia: A new material for posteriors with minimal tooth preparation. Adv Human Biology 2014; 4(1): 31-36.

[3]. Gurav SV, Ram SM, Khanna TS. Occlusal rehabilitation of severely mutilated dentition. J Contemp Dent Jan Apr 2015; 5 (1): 4852.

[4]. Mete JJ, Dange SP, Khalikar AN, Vaidya SP. Functional and esthetic rehabilitation of mutilated dentition associated with amelogenesis imprefecta. J Indian Prosthodont Soc. 2012 Jun; 12 (2): 94-100.

[5]. Dua P, Singh J.P, Aghi A. Aesthetic and functional rehabilitation of a case of mutilated dentition and loss of vertical dimensions. J Indian Prostho Dont Soc. 2011 Sep; 11 (3): 189-194.

[6]. Wankhade S.V, Lokade J, rajguru V. Prosthodonic rehabilitation of mutilated dentition. Int J Dent Clin 2011: 3 (2): $103-104$.

[7]. Mittal S, Tewari S, Goel R. Esthetic and functional rehabilitation of mutilated dentition and loss of vertical dimension due to amelogenesis imperfecta. Ind J Dent 2014; 5(2): 102-106.

[8]. Ambulekar J, Sanadi R, Doshi M, Gaikwad S. Full mouth rehabilitation of mutilated dentition - a case report. Int J Innov Res Sci, Eng, Tech. 2013 Aug; 2 (8): 3697-3700.

[9]. Thumati P, Reddy R, Padmaja S. Multidisciplinary approach in full mouth rehabilitation- from ruins to riches in oral health. J Dent Med Scie 2013 Nov-Dec; 12 (3): 25-29. 